
n. 4, p.597-600, dezembro 2002.

\title{
Aplicações de fertilizantes foliares na nutrição e na produção do pimen- tão e do tomateiro
}

\author{
Hamilton S. Pereira ${ }^{1}$; Simone C. Mello ${ }^{2}$ \\ ${ }^{1}$ UFU, Depto. Agronomia, C. Postal 593, 38.400-902 Uberlândia-MG; ${ }^{2}$ Centro Universitário Moura Lacerda, 1520, 14.076-510 Ribeirão \\ Preto-SP; E-mail: mello.simone@ig.com.br
}

\section{RESUMO}

Avaliou-se a eficiência de fertilizantes foliares, contendo Ca e $\mathrm{B}$, na nutrição e produção do pimentão cv. Mayata e do tomate cv. Carmem, cultivados em estufas. O delineamento experimental foi de blocos ao acaso com seis tratamentos, três e quatro repetições, respectivamente, para o tomateiro e pimentão. Os tratamentos foram a água de retortagem de xisto $(100 ; 78 ; 3,0 ; 12,5 ; 3,0 ; 12,5 ; 4,0$ e 0,2 g.L.- de N, K, S, Ca, Mg, B, Mn e Mo, respectivamente) a 0,1; 0,$25 ; 0,5$ e $1 \%$; os sais ácido bórico ( 170 g.kg ${ }^{-1}$ de B) a $0,15 \%$ mais cloreto de cálcio a $0,15 \%$ e o quelato de cálcio $\left(200 \mathrm{~g} \cdot \mathrm{kg}^{-1} \mathrm{de}\right.$ Ca) a $0,1 \%$ e boro orgânico $\left(10 \mathrm{~g} \cdot \mathrm{kg}^{-1} \mathrm{de} \mathrm{B}\right)$ a $0,15 \%$. A aplicação de água de retortagem de xisto a $0,5 \%$ via foliar aumentou em $27,4 \%$ a produção de frutos de pimentão e os teores foliares de $\mathrm{Mg}$ e $\mathrm{S}$ em relação à aplicação desse fertilizante a $0,1 \%$. Para o tomateiro, as pulverizações com água de retortagem de xisto a 0,5 e $1 \%$ elevaram em 60,5 e $93,8 \%$ a produção de frutos em comparação à menor dose desse fertilizante. Os maiores teores de B nas folhas de pimentão e de tomate foram obtidos com as aplicações de sais e resultaram em sintomas visuais de toxicidade de $\mathrm{B}$ no início do desenvolvimento das culturas.

Palavras-chave: Capsicum annuum, Lycopersicon esculentum, cálcio, boro, água de retortagem de xisto.

\begin{abstract}
Foliar fertilizer applications on nutrition and yield of sweet pepper and tomato

The efficiency of foliar fertilizers was evaluated on nutrition and yield of sweet pepper cv. Mayata and tomato cv. Carmem, cultivated in plastic greenhouse. For the two experiments the experimental design consisted of randomized blocks with three and four replications for tomato and sweet pepper, respectively, and six treatments (schist retortage water $0.1 ; 0.25 ; 0.5$ and $1 \%$; boric acid $0.15 \%$ plus clorate calcium $0.15 \%$ and quelate $\mathrm{Ca} 0.1 \%$ plus organic B $0.15 \%$ ). The foliar application of schist retortage water $0.5 \%$ increased $27.4 \%$ the sweet pepper fruit yield and foliar concentrations of $\mathrm{Mg}$ and $\mathrm{S}$ compared to this fertilizer $0.1 \%$. For tomato, the application of schist retortage water 0.5 and $1 \%$ increased 60.5 and $93.8 \%$ the fruit yield as compared to this fertilizer $0.1 \%$. The higher levels of boron in sweet pepper leaves and in tomato leaves were obtained with salt applications and resulted in visual symptoms of boron toxicity on initial development of cultures.
\end{abstract}

Keywords: Capsicum annuum, Lycopersicon esculentum, calcium, boron, schist retortage water.

\section{(Recebido para publicação em 21 de março de 2001 e aceito em 20 de maio de 2002)}

A aplicação de fertilizantes foliares tem se desenvolvido rapidamente nos últimos anos, não só no exterior como também no Brasil, em virtude, dentre outros fatores, da necessidade de se buscar altas produtividades das culturas. Neste contexto, produtos cada vez mais eficientes e econômicos têm sido desenvolvidos para satisfazer as exigências nutricionais das plantas. Atualmente um grande número de fertilizantes foliares está disponível no mercado, como fornecedor de um ou mais elementos essenciais, sendo que dentre eles os sais e os quelatos são os mais comumente utilizados pelos agricultores.

$\mathrm{Na}$ cultura do tomateiro (Lycopersicon esculentum Mill) e do pimentão (Capsicum annuum L.), espécies olerícolas pertencentes à família Solanaceae, de alto valor econômico, a aplicação de fertilizantes foliares, incluindo o $\mathrm{Ca}$ e o B, tem sido praticada pelos produtores, visando o aumento de produção e a qualidade dos frutos. Entretanto, a escassez de pesquisas no Brasil, sobre os efeitos de diferentes formas e fontes desses micronutrientes no desenvolvimento de tais hortaliças, requer novos estudos, principalmente em condições de ambiente protegido, onde o cultivo delas vem aumentando cada vez mais.

Para o tomateiro, na prevenção da podridão apical, causada pelo fornecimento inadequado de $\mathrm{Ca}$, Castellane (1988) recomenda pulverizações foliares com $\mathrm{CaCl}_{2}$ 0,6\% semanalmente, a partir do ínicio do florescimento das plantas, dirigidas para os frutos. Ainda, a sua aplicação via foliar pode incrementar a produção de tomate. Csizinszky (1994) obteve maior produção de tomate cv. Agriset 761 com quatro aplicações foliares de fertilizante orgânico contendo 3-6 g.L. $\mathrm{L}^{-1} \mathrm{~N}$; 1,3-2,6 g.L ${ }^{-1} \mathrm{P} ; 33-51$ g.L ${ }^{-1} \mathrm{~K} ; 3-6$ g.L $\mathrm{L}^{-1} \mathrm{~S} ; 1-2$
g.L.1 Mg; 0,5-1,0 g.L.-1 Ca; 44-75 mg.L${ }^{1} \mathrm{Fe}$; 9-15 mg.L ${ }^{-1} \mathrm{Cu}$; $15-23 \mathrm{mg} . \mathrm{L}^{-1} \mathrm{Zn}$; 2-4 mg.L. $\mathrm{L}^{-1} \mathrm{Mn}$ e $30-44 \mathrm{mg} . \mathrm{L}^{-1} \mathrm{~B}$, juntamente com 292,5 e 486,5 kg.ha-1 de N e $\mathrm{K}$ via solo, respectivamente, em relação à ausência de fertilizante foliar, porém com aplicação de $195 \mathrm{~kg} \cdot \mathrm{ha}^{-1}$ de N e 324 kg.ha-1 de K via solo.

Em relação ao B, a sua aplicação via foliar tem mostrado resultados positivos no desenvolvimento do tomateiro. Magalhães \& Monnerat (1978) observaram aumento de produção de matéria seca das raízes e da parte aérea de plantas de tomate com a aplicação foliar de ácido bórico a 1\%, em intervalos de três e seis dias de pulverização. Csizinszky (1984), estudando a aplicação de fertilizante orgânico via foliar nessa cultura, verificou que as pulverizações com 2,5 L.ha-1 de MZ63 (41,7 N; $30 \mathrm{Mg}$; 46,8 S; 8,1

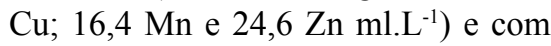
2,5 L.ha-1 de BM 86 (41,7 N; 30 Mg; 25 


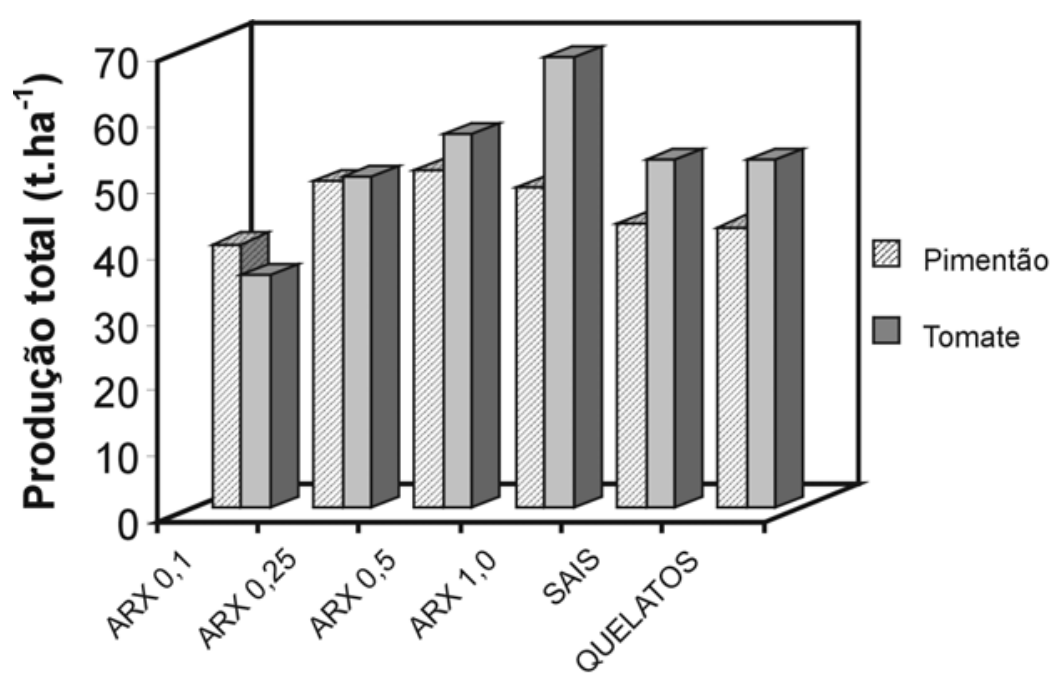

Fertilizantes foliares

Figura 1. Efeito de fertilizantes foliares na produção total de pimentão cv. Mayata e de tomate cv. Carmem. Piracicaba, 1995 e 1996.

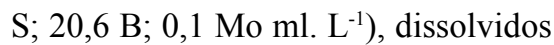
em 200 a $378 \mathrm{~L}$ de $\mathrm{H}_{2} \mathrm{O}$, aumentaram em $74 \%$ a produção de frutos comerciáveis de tomate cv. Sunny. Por outro lado, Csizinszky (1986) obteve redução na produção comerciável de tomate com duas aplicações de MZ63 e duas aplicações de BM86. Em plantas de pimentão, aplicações foliares semanais com os biofertilizantes Supermagro (2; $4 ; 6 ; 8$ e $10 \%)$ e Biofertilizante bovino $(10 ; 20 ; 30 ; 40$ e $50 \%)$ não aumentaram a produção total de frutos em relação à testemunha (Souza, 2000).

Pode-se notar, que para a cultura do pimentão a necessidade de se obter informações sobre os efeitos de fertilizantes foliares, contendo $\mathrm{Ca}$ e $\mathrm{B}$, no desenvolvimento das plantas é ainda maior. Dessa forma, realizou-se esse trabalho com o objetivo de comparar a água de retortagem de xisto betuminoso (resíduo da mineração e industrialização do xisto betuminoso) com sais de $\mathrm{Ca}$ e B, quelato de $\mathrm{Ca}$ com B orgânico na nutrição e produção do pimentão e do tomateiro, em estufa.

\section{MATERIAL E MÉTODOS}

Dois experimentos foram conduzidos no município de Piracicaba, de junho de 1995 a fevereiro de $1996 \mathrm{em} \mathrm{um}$ Podzólico vermelho-amarelo abrupto, eutrófico, A moderado, textura arenosa/ média, cujas características químicas fo- ram: $\mathrm{pH}\left(\mathrm{CaCl}_{2}\right)$ 5,2; M.O. $\left(\right.$ g.dm $\left.{ }^{-3}\right) 15$; $\mathrm{P}\left(\mathrm{mg} \cdot \mathrm{dm}^{-3}\right) 111 ; \mathrm{K}\left(\mathrm{mmol}_{\mathrm{c}} \cdot \mathrm{dm}^{-3}\right)$ 1, 7 ; Ca $\left(\mathrm{mmol}_{\mathrm{c}} \cdot \mathrm{dm}^{-3}\right)$ 29; $\mathrm{Mg}\left(\mathrm{mmol}_{\mathrm{c}} \cdot \mathrm{dm}^{-3}\right)$ 14; $\mathrm{H}+\mathrm{Al}\left(\mathrm{mmol}_{\cdot} \cdot \mathrm{dm}^{-3}\right) 20$.

As mudas de pimentão cv. Mayata e de tomate cv. Carmem foram produzidas em bandejas de 128 células e transplantadas para estufas, 25 dias após a germinação das sementes. As estufas apresentavam estrutura metálica, na forma de arco com $7 \mathrm{~m}$ de largura por 60 $m$ de comprimento e altura do vão central de $4,5 \mathrm{~m}$, cobertas com plástico transparente (200 micras de espessura e com aditivo contra os raios ultra violeta) e com as laterais fechadas com tela de sombrite preto a $30 \%$.

Realizou-se antes do plantio, em área total, adubação fosfatada, empregandose 320 e $450 \mathrm{~kg}$. ha-1 de $\mathrm{P}_{2} \mathrm{O}_{5}$ na forma de superfosfato simples, nos locais referentes ao plantio de pimentão e de tomate, respectivamente. $\mathrm{O}$ nitrogênio e o potássio foram aplicados via fertirrigação (Tabela 1). Quinze dias após o transplante das mudas iniciou-se a aplicação dos fertilizantes foliares, semanalmente, até o final da colheita.

O delineamento adotado para ambos os experimentos foi de blocos ao acaso com seis tratamentos, três e quatro repetições, respectivamente, para o tomateiro e para o pimentão. Os tratamentos foram a água de retortagem de xisto $(100 ; 78 ; 3,0 ; 12,5 ; 3,0 ; 12,5 ; 4,0$ e 0,2 g. $\mathrm{L}^{-1}$ de N, K, S, Ca, Mg, B, Mn e Mo, respectivamente) a 0,$1 ; 0,25 ; 0,5$ e $1 \%$, os sais ácido bórico (170 g. $\left.\mathrm{kg}^{-1} \mathrm{de} \mathrm{B}\right) \mathrm{a}$ $0,15 \%$ mais cloreto de cálcio a $0,15 \%$ e o quelato de cálcio (200 g.kg-1 de Ca) a $0,1 \%$ com boro orgânico (10 g. $\mathrm{kg}^{-1}$ de B) a $0,15 \%$.

$\mathrm{O}$ transplante do pimentão foi realizado em linha dupla no espaçamento de $0,35 \mathrm{~m}$ entre plantas e $0,4 \mathrm{~m}$ entre linhas, com parcela de $6 \mathrm{~m}$ de comprimento e 16 plantas. Para o tomateiro, adotou-se o espaçamento de $0,6 \mathrm{~m}$ entre plantas e $1,0 \mathrm{~m}$ entre linhas, com parcelas de uma linha de $6 \mathrm{~m}$ de comprimento intercaladas com linhas não tratadas (bordadura).

No período do aparecimento dos primeiros frutos maduros, foram realizadas as amostragens de folhas, coletando-se a primeira folha de pimentão logo abaixo da segunda flor aberta e a folha de tomate logo abaixo e oposta ao segundo cacho floral para a determinação dos teores de macronutrientes e dos micronutrientes $\mathrm{B}, \mathrm{Cu}, \mathrm{Fe}, \mathrm{Mn}$ e $\mathrm{Zn}$ (Malavolta et al., 1989).

Tratos culturais como capinas, desbrotas, controle de pragas e doenças foram feitos de acordo com as necessidades das culturas. As colheitas dos frutos foram feitas semanalmente para a obtenção da produção total.

\section{RESULTADOS E DISCUSSÃO}

A produção de frutos de pimentão e de tomate foi significativamente influenciada $(\mathrm{P}<0,05)$ pelos fertilizantes foliares (Figura 1). A aplicação de água de retortagem de xisto a $0,5 \%$ aumentou em $27,4 \%$ a produção de pimentão $\left(51,3\right.$ t.ha $\left.^{-1}\right)$ em relação à aplicação desse fertilizante a $0,1 \%\left(40,2\right.$ t.ha $\left.{ }^{-1}\right)$. Souza (2000) não constatou aumento na produção de frutos de pimentão com as aplicações foliares semanais de Supermagro $(2 ; 4 ; 6 ; 8$ e $10 \%)$ ou de Biofertilizante $(10 ; 20 ; 30 ; 40$ e $50 \%)$.

Sintomas visuais de toxicidade de B foram observados com as aplicações de sais e quelato de Ca com B orgânico, caracterizados por queimaduras das bordas das folhas, no período inicial do desenvolvimento da cultura. Estes resultados foram coerentes com os teores foliares de $\mathrm{B}$, bem mais elevados para 
Tabela 1. Adubação nitrogenada e potássica via fertirrigação para as áreas de $336 \mathrm{~m}^{2}$ (áreas das estufas) cultivadas com tomate e pimentão. Piracicaba, 1995 e 1996.

\begin{tabular}{|c|c|c|c|c|}
\hline \multirow{3}{*}{ Dias } & \multicolumn{2}{|c|}{ Pimentão } & \multicolumn{2}{|c|}{ Tomate } \\
\hline & $\mathrm{NH}^{4} \mathrm{NO}^{3}$ & $\mathrm{KCl}$ & $\mathrm{NH}_{4} \mathrm{NO}_{3}$ & $\mathrm{KCl}$ \\
\hline & \multicolumn{4}{|c|}{ g $336 \mathrm{~m}^{2} \cdot \mathrm{dia}^{-1}$} \\
\hline $1-10$ & 10 & 5 & 15 & 7 \\
\hline $11-20$ & 22 & 7 & 35 & 10 \\
\hline $21-30$ & 50 & 7 & 75 & 10 \\
\hline $31-40$ & 85 & 15 & 125 & 20 \\
\hline $41-50$ & 140 & 235 & 205 & 400 \\
\hline $51-60$ & 170 & 235 & 250 & 400 \\
\hline $61-70$ & 170 & 235 & 250 & 400 \\
\hline $71-80$ & 185 & 330 & 275 & 490 \\
\hline $81-90$ & 240 & 330 & 350 & 490 \\
\hline $91-100$ & 340 & 350 & 500 & 520 \\
\hline $101-110$ & 400 & 350 & 600 & 520 \\
\hline $111-120$ & 340 & 360 & 500 & 550 \\
\hline $121-130$ & 340 & 50 & 500 & 70 \\
\hline $131-150$ & 200 & 50 & 300 & 70 \\
\hline $150-200$ & 100 & 50 & 150 & 70 \\
\hline
\end{tabular}

Tabela 2. Teores foliares de nutrientes em plantas de pimentão cv. Mayata. Piracicaba, 1995 e 1996.

\begin{tabular}{|c|c|c|c|c|c|c|c|c|c|c|}
\hline \multirow{2}{*}{ Trat } & $\mathbf{N}$ & $\mathbf{P}$ & K & $\mathrm{Ca}$ & Mg & $S$ & B & $\mathrm{Cu}$ & $M n$ & $\mathrm{Zn}$ \\
\hline & \multicolumn{6}{|c|}{ g. $\mathbf{k g}^{-1}$} & \multicolumn{4}{|c|}{ mg.kg ${ }^{-1}$} \\
\hline ARX 0,1\% & 34,9 & 2,0 & 66,9 & 37,9 & $5,4 b$ & $3,1 b$ & $95 \mathrm{c}$ & 83 & $261 \mathrm{c}$ & 162 \\
\hline ARX $0,25 \%$ & 34,7 & 2,0 & 69,4 & 43,4 & $5,8 a b$ & $3,4 a b$ & $98 c$ & 89 & 293 bc & 122 \\
\hline ARX 0,5\% & 35,4 & 2,0 & 69,6 & 45,6 & $6,5 a$ & $3,9 a$ & $123 \mathrm{bc}$ & 97 & $390 \mathrm{ab}$ & 206 \\
\hline ARX $1,0 \%$ & 34,9 & 2,1 & 72,2 & 38,9 & $5,9 a b$ & $3,8 a$ & $165 \mathrm{~b}$ & $11 \mathrm{a}$ & $445 a$ & 423 \\
\hline Sais & 33,1 & 2,0 & 69,3 & 40,0 & $5,5 a b$ & $3,4 a b$ & $347 \mathrm{a}$ & 87 & $274 \mathrm{c}$ & 126 \\
\hline Quelatos & 32,6 & 1,9 & 69,7 & 39,6 & $5,9 a b$ & $3,4 a b$ & $311 \mathrm{a}$ & 86 & $285 \mathrm{c}$ & 133 \\
\hline$\overline{\mathrm{CV} \%}$ & 6,5 & 6,1 & 5,8 & 8,7 & 7,5 & 6,5 & 12,6 & 45,1 & 13,2 & 69,2 \\
\hline DMS & 5,2 & 0,3 & 9,3 & 8,2 & 1,0 & 0,5 & 54,9 & 96,7 & 98,3 & 311,5 \\
\hline
\end{tabular}

os tratamentos com sais e quelato de $\mathrm{Ca}$ com B orgânico (Tabela 2).

Além do B, os teores de $\mathrm{Mg}, \mathrm{S}$ e $\mathrm{Mn}$ nas folhas de pimentão foram alterados pelos tratamentos empregados (Tabela 2). Os teores de $\mathrm{Mg}$ e $\mathrm{S}$ nas folhas foram maiores com a adição de água de retortagem de xisto a $0,5 \%$ em relação a menor dose desse fertilizante, corroborando com os resultados obtidos para a produção. As concentrações foliares de Mn foram superiores com as aplicações de água de retortagem de xisto nas maiores doses quando comparadas à menor dose desse fertilizante, aos sais e quelato de Ca com B orgânico.
Para o tomateiro, verifica-se que as aplicações de água de retortagem de xisto a 0,5 e $1 \%$ aumentaram a produção de frutos em comparação à menor dose desse fertilizante. Csizinszky (1994), no entanto, não observou diferença significativa entre quatro e oito aplicações foliares de um fertilizante orgânico na produção de frutos de tomate. Conforme observado para o pimentão, a água de retortagem de xisto não foi superior aos sais e quelatos de Ca mais B orgânico. O tratamento com sais, por sua vez, também foi responsável pelo aparecimento de sintomas visuais de toxicidade, observados através da queima leve das bordas das folhas, no início do desenvolvimento da cultura. Este fato está de acordo com o maior teor foliar de B nas plantas que receberam a aplicação de sais (Tabela 3).

Com exceção do $\mathrm{B}$ e do $\mathrm{Ca}$, os demais teores dos nutrientes nas folhas não sofreram influência das aplicações dos fertilizantes foliares (Tabela 3). O maior teor de Ca foi obtido com a aplicação da água de retortagem de xisto a $1,0 \%$ em relação a este fertilizante a $0,25 \%$, não havendo diferença significativa entre os demais tratamentos.

$\mathrm{O}$ aumento no teor de B em folhas de tomate com as aplicações dos sais não interferiu no desempenho produtivo da 
Tabela 3. Teores foliares de nutrientes em plantas de tomate cv. Carmem. Piracicaba, 1995 e 1996.

\begin{tabular}{|c|c|c|c|c|c|c|c|c|c|c|}
\hline \multirow{2}{*}{ Trat } & $\mathbf{N}$ & $\mathbf{P}$ & $\mathrm{K}$ & $\mathrm{Ca}$ & $\mathrm{Mg}$ & $\mathbf{S}$ & B & $\mathrm{Cu}$ & $\mathrm{Mn}$ & $\mathrm{Zn}$ \\
\hline & \multicolumn{6}{|c|}{ g. $\mathbf{k g}^{-1}$} & \multicolumn{4}{|c|}{ mg.kg ${ }^{-1}$} \\
\hline ARX $0,1 \%$ & 25,7 & 3,7 & 46,6 & $35,6 a b$ & 7,6 & 4,3 & $71 \mathrm{c}$ & 4,67 & 164 & 50 \\
\hline ARX 0,25\% & 26,8 & 3,4 & 45,3 & $31,7 b$ & 6,5 & 4,7 & $73 \mathrm{c}$ & 8,33 & 154 & 77 \\
\hline ARX 0,5\% & 30,7 & 3,7 & 48,8 & $40,6 a b$ & 7,6 & 4,8 & $85 \mathrm{bc}$ & 4,33 & 234 & 128 \\
\hline$A R X 1,0 \%$ & 24,7 & 4,0 & 42,8 & $45,3 a$ & 6,5 & 5,6 & $106 \mathrm{bc}$ & 6,67 & 251 & 102 \\
\hline Sais & 30,7 & 4,0 & 48,5 & $41,5 a b$ & 7,0 & 4,5 & $189 a$ & 4,00 & 171 & 136 \\
\hline Quelatos & 28,0 & 4,1 & 43,4 & $36,2 a b$ & 6,5 & 4,5 & $141 \mathrm{ab}$ & 4,00 & 138 & 60 \\
\hline CV \% & 15,6 & 14,9 & 6,78 & 11,1 & 17,2 & 11,5 & 20,5 & 51,1 & 21,7 & 36,6 \\
\hline DMS & 12,3 & 01,5 & 8,8 & 12,1 & 3,7 & 1,5 & 64,31 & 7,72 & 114,5 & 95,7 \\
\hline
\end{tabular}

cultura. Resultados discordantes foram constatados por Csizinszky (1986), onde os maiores teores de B (154 mg.kg $\left.{ }^{-1}\right)$, $\mathrm{Cu}, \mathrm{Fe}, \mathrm{Mn}$ e $\mathrm{Zn}$, resultantes das aplicações dos biofertilizantes foliares MZ63 e BM86, refletiram em elevação da produção em relação ao biofertilizante Keyplex 350 e ao controle.

Com exceção do $\mathrm{N}$ e do $\mathrm{Mg}$, os teores foliares de $\mathrm{K}, \mathrm{Ca}, \mathrm{B}, \mathrm{Cu}, \mathrm{Mn}$ e $\mathrm{Zn}$ estão, de modo geral, acima das faixas de concentrações consideradas adequadas para a cultura do pimentão, sendo que os níveis de fósforo foram inferiores ao limite mínimo adequado (Trani \& Raij, 1996). Para o tomateiro, apenas os teores de $\mathrm{N}$ nas folhas estão abaixo da faixa adequada, sendo que os teores foliares de $\mathrm{B}$ pertencentes à maior dose de água de retortagem de xisto, aos sais e ao quelato de Ca com B orgânico são superiores aos valores indicados pelos autores acima.

Os resultados obtidos permitem inferir que a água de retortagem de xisto pode ser utilizada como fertilizante foliar para as plantas de pimentão e de tomate. Este fato significa redução no custo de produção e viabilidade agronômica do resíduo da mineração e da industrialização do xisto betuminoso.

\section{LITERATURA CITADA}

CASTELLANE, P.D. Podridão apical em frutos de tomateiro. Jaboticabal: FUNEP, 1988. 39 p.

CSIZINSZKY, A.A. Response of tomatoes to foliar biostimulant sprays. Proceedings of the Florida State Horticultural Society, v. 99, p. 353-358, 1986.

CSIZINSZKY, A.A. Response of tomatoes to seaweed based nutrient sprays. Proceedings of the Florida State Horticultural Society, v. 97, p. 151157, 1984.
CSIZINSZKY, A.A. Yield response of tomato, cv. Agriset 761, to seaweed spray, micronutrient, and $\mathrm{N}$ and K rates. Proceedings of the Florida State Horticultural Society, v. 107, p. 139-142, 1994. MAGALHÃES, J.R.; MONNERAT, P.H. Aplicação foliar de boro na prevenção de deficiência e na composição mineral do tomateiro. Pesquisa Agropecuária Brasileira, Brasília, v. 13, p. 73-80, 1978.

MALAVOLTA, E.; VITTI, G.C.; OLIVEIRA, S.A. Avaliação do estado nutricional das plantas, princípios e aplicações. Piracicaba: POTAFOS, 1989. $201 \mathrm{p}$.

SOUZA, J.L. Nutrição orgânica com biofertilizantes foliares na cultura do pimentão em sistema orgânico. Horticultura Brasileira, Brasília, v. 18, p. 828-929, 2000 (Suplemento).

TRANI, P.E.; RAIJ, B. van. Hortaliças. In: INSTITUTO AGRONÔMICO DE CAMPINAS. Recomendações de adubação e calagem para o Estado de São Paulo. Campinas, 1996. p. 155-203. (IAC. Boletim Técnico, 100). 